\title{
NE ESIS
}

Negative Effects in Medical Sciences Oral and Maxillofacial Surgery

\section{UCL PRESSES}

\section{Déplacement accidentel de la dent de sagesse maxillaire dans le vestibule jugal antérieur}

\author{
Authors: \\ Fathi S DDS ${ }^{1, *}$ \\ Olszewski R DDS, MD, PhD, DrSc, Prof ${ }^{2,3}$
}

\author{
Affiliations: \\ ${ }^{1}$ Polyclinique du val de Sambre, Stomatologie, Route de Mons 162, 59600 \\ Maubeuge, France \\ ${ }^{2}$ Oral and maxillofacial surgery research Lab, NMSK, IREC, SSS, UCLouvain, \\ Brussels, Belgium \\ ${ }^{3}$ Department of oral and maxillofacial surgery, Cliniques universitaires saint Luc, \\ UCLouvain, Brussels, Belgium \\ *Corresponding author: Fathi S, Docteur.s.fathi@gmail.com, ORCID Id: 0000 \\ 0001-9439-671X \\ Disclaimer: the views expressed in the submitted article are our own and not an \\ official position of the institution or funder.
}




\section{Résumé}

Les déplacements accidentels des dents de sagesse du maxillaire supérieur dans divers régions anatomiques sont rares. Nous avons effectué la recherche de littérature sur ce sujet de manière systématique en utilisant PubMed et DOAJ. Il n'existe pas d'illustration accessible gratuitement pour les voies de déplacements accidentels des dents de sagesse supérieurs imagées par le CT scan ou par le CBCT à part le déplacement vers la fosse infra-temporale et vers la fosse ptérygopalatine. Nous décrivons et illustrons par $\mathrm{CBCT}$ un cas unique dans la littérature médicale de déplacement accidentel du germe de la dent de sagesse du maxillaire supérieur dans l'espace jugal antérieur. Les raisons potentielles, les conséquences ainsi que les moyens de prévention de cette rare complication d'extraction de dents de sagesse sont aussi expliquées.

\section{Abstract}

Accidental displacements of the wisdom teeth of the upper jaw to various anatomical regions are rare. We systematically searched the literature on this issue using PubMed and DOAJ. There is no freely available illustration for the accidental displacement pathways of the upper wisdom teeth imaged by CT scan or by CBCT apart from displacement towards the infra-temporal, and the pterygopalatine fossa. We describe and illustrate by CBCT a unique case in the medical literature of accidental displacement of the wisdom tooth germ of the upper jaw into the anterior jugal space. The potential reasons, consequences and ways to prevent this rare complication of wisdom tooth extraction are also explained.

Keywords: third molar displacement, wisdom teeth displacement, CBCT 


\section{Introduction}

Les déplacements accidentels des dents de sagesse du maxillaire supérieur dans divers régions anatomiques sont rares [1,2]. Nous avons procédé d'abord à la recherche de la littérature médicale sur ce sujet de manière systématique. Nous avons retenu deux bases de données gratuites: PubMed et DOAJ (Directory of open access journals) et nous avons réalisé la recherche en dehors des serveurs universitaires pour se retrouver dans les mêmes conditions d'accès à aux articles scientifiques qu'un praticien dentiste privé. Un observateur a réalisé la recherche des articles. Les critères d'inclusion étaient: dents de sagesse ou troisième molaire, maxillaire supérieur, déplacement accidentel. Nous avons retenus tous les cas cliniques y compris des descriptions basées sur un seul patient. Nous avons retenus 2 langues: Anglais et Français et des articles avec abstract. Les critères d'exclusion étaient les dents de sagesse en position ectopique, les dents de sagesse mandibulaires déplacés accidentellement, des articles sans abstract et publiés dans d'autres langues que l'anglais et le français. Nous avons proposé 2 équations de recherche dans PubMed:

1. (("molar, third"[MeSH Terms] OR ("molar"[All Fields] AND "third"[All Fields]) OR "third molar"[All Fields] OR ("third"[All Fields] AND "molar"[All Fields])) AND ("displace"[All Fields] OR "displaced"[All Fields] OR "displacement, psychological"[MeSH Terms] OR ("displacement"[All Fields] AND "psychological"[All Fields]) OR "psychological displacement"[All Fields] OR "displacement"[All Fields] OR "displacements"[All Fields] OR "displaces"[All Fields] OR "displacing"[All Fields]) AND ("maxilla"[MeSH Terms] OR "maxilla"[All Fields] OR "maxillae"[All Fields] OR "maxillas"[All Fields])) AND (english[Filter] OR french[Filter]). Cette recherche a eu lieu le 13.03.2021 et a donné 39 articles. 12 articles ont été retenus [3-14] pour la revue de la littérature et 27 articles ont été exclus selon les critères d'exclusion choisis.

2. (("molar, third"[MeSH Terms] OR ("molar"[All Fields] AND "third"[All Fields]) OR "third molar"[All Fields] OR ("wisdom"[All Fields] AND "teeth"[All Fields]) OR "wisdom teeth"[All Fields]) AND ("displace"[All Fields] OR "displaced"[All Fields] OR "displacement, psychological"[MeSH Terms] OR ("displacement"[All Fields] AND "psychological"[All Fields]) OR "psychological displacement"[All Fields] OR "displacement"[All Fields] OR "displacements"[All Fields] OR " displaces"[All Fields] OR "displacing"[All Fields]) AND ("maxilla"[MeSH Terms] OR "maxilla"[All Fields] OR "maxillae"[All Fields] OR "maxillas"[All Fields])) AND (english[Filter] OR french[Filter]). Cette recherche a eu lieu le 13.03.2021 et a donné 40 articles. 13 articles ont été retenus. 27 articles ont été exclu selon les critères d'exclusion établis. Cependant 12 articles étaient identiques par rapport à la $1^{\text {ère }}$ équation et finalement un seul article a été retenu [15]. 
Nous avons également abordé cette recherche de littérature sous l'aspect de l'accès ouvert à la littérature scientifique. Nous avons proposé une équation de recherche dans Pubmed en sélectionnant les articles avec l'accès ouvert à l'article et dans la base de données DOAJ (mots-clés : « third molar », « displacement »)

Pubmed : (("molar, third"[MeSH Terms] OR ("molar"[All Fields] AND "third"[All Fields]) OR "third molar"[All Fields] OR ("third"[All Fields] AND "molar"[All Fields])) AND ("displace"[All Fields] OR "displaced"[All Fields] OR

"displacement, psychological"[MeSH Terms] OR ("displacement"[All Fields] AND "psychological"[All Fields]) OR "psychological displacement"[All Fields] OR " displacement"[All Fields] OR "displacements"[All Fields] OR "displaces"[All Fields] OR "displacing"[All Fields]) AND ("maxilla"[MeSH Terms] OR "maxilla"[All Fields] OR "maxillae"[All Fields] OR "maxillas"[All Fields])) AND ((ffrft[Filter]) AND (english[Filter] OR french[Filter])). Cette recherche a eu lieu le 13.03.2021 et a donné 6 articles. Aucun de ces articles ne fut retenu par rapport aux critères d'inclusion établis.

DOAJ :

https://doaj.org/search/articles?ref=homepagebox\&source $=\% 7 \mathrm{~B} \% 22$ query $\% 22 \% 3 \mathrm{~A} \% 7 \mathrm{~B} \% 22$ query string $\% 22 \% 3 \mathrm{~A} \% 7 \mathrm{~B} \% 22$ quer $\mathrm{y} \% 22 \% 3 \mathrm{~A} \% 22$ third $\% 20$ molar $\% 20$ displacement $\% 20 \% 22 \% 2 \mathrm{C} \% 22$ default operator $\% 22 \% 3 \mathrm{~A} \% 22 \mathrm{AND} \% 22 \% 2 \mathrm{C} \% 22$ default field $\% 22 \% 3 \mathrm{~A} \% 22$ bibjson.abstract $\% 22 \% 7$ $\mathrm{D} \% 7 \mathrm{D} \% 7 \mathrm{D}$

Cette recherche a eu lieu le 13.03.2021 et a donné 26 articles en utilisant les mots clés : « third molar » et « displacement ». 4 articles ont été retenus [16-19] et 22 articles ont été exclus par rapport aux critères d'inclusion établis.

Au total, cette revue de littérature médicale se base sur 13 abstracts [3-15] et 4 articles [16-19] accessibles en accès ouvert (pdf gratuit pour les lecteurs). Le déplacement accidentel de la dent de sagesse au maxillaire supérieur peut se faire vers diverses régions anatomiques (Figure 1) : 1) la fosse infra-temporale avec le plus de cas publiés dans la littérature $[4,5,8,10,11,14,16,17]$;2) le sinus maxillaire $[12,15]$; 3) l'espace latéro-pharyngé [7];4) la région de la tubérosité maxillaire [18] ; 5) la fosse ptérygopalatine [19]; et 6) l'espace buccal (boule de Bichat) $[6,9]$. 


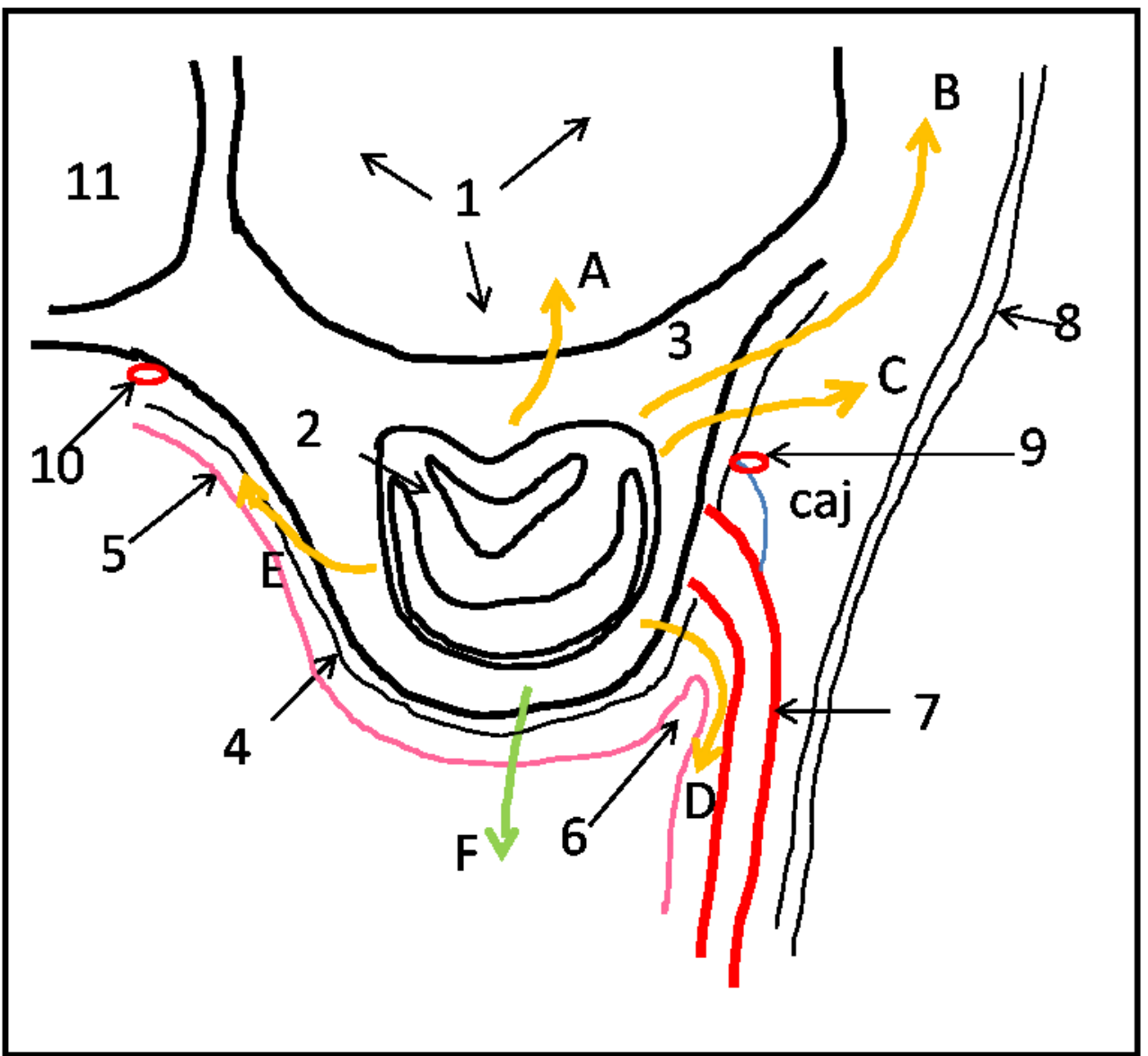

Fig. 1. 1. Sinus maxillaire. 2. Germe de la dent de sagesse du maxillaire supérieur. 3. Maxillaire supérieur. 4. Périoste. 5. Muqueuse palatine. 6. Vestibule jugal. 7. Muscle buccinateur. 8. Peau. 9. Artère alvéolaire supérieure et postérieure. 10. Vaisseaux palatins. 11. Cavité nasale. caj: corps adipeux de la joue (anciennement boule de Bichat).

A. Déplacement du germe dans le sinus maxillaire. B. Déplacement du germe dans la fosse infra-temporale. C. Déplacement du germe dans le corps adipeux de la joue (espace jugal postérieur). D. Déplacement du germe dans l'espace jugal antérieur (le cas décrit). E. Déplacement du germe au palais.

Les symptômes dépendent de la localisation de la dent déplacée. S'il s'agit d'un déplacement de la dent de sagesse dans la fosse infra-temporale, le patient présentera un œdème facial unilatéral, la douleur et un trismus sévère [4]. Dans le cadre de déplacement vers la fosse ptérygopalatine le patient présentera un léger œè̀me 
facial, un trismus sévère et la dent de sagesse ne sera pas palpable dans les tissus mous [19].

L'examen complémentaire de choix est actuellement le CBCT pour la localisation exacte de la dent $[16,18]$.

Les raisons du déplacement accidentel de la dent de sagesse maxillaire invoqués dans la littérature médicale sont : un examen clinique et radiologique insuffisants, les connaissances anatomiques insuffisantes, un lambeau inadéquat [16], la faible visibilité du site opératoire et de la dent à luxer, une force excessive, non-contrôlée sur l'élévateur pendant le geste chirurgical [19].

En cas de passage dans la fosse infra-temporale il est recommandé de ne pas intervenir immédiatement du fait des risques hémorragiques (artère maxillaire), neurologique (nerf sous-orbitaire) et d'un déplacement supplémentaire de la dent vers postérieur et crânial [11]. Le patient doit être mis sous antibiotiques [13] et dirigé vers un service de chirurgie maxillo-faciale [11].

Les traitements proposés en cas de déplacement accidentel de la dent de sagesse dans la fosse infra-temporale [1] sont variables: 1) endoscopie transorale de la fosse infratemporale [2], 2) passage trans-sinus maxillaire de type Caldwell-Luc [17], 3) approche à travers le sinus maxillaire avec la trépanation du mur postérieur du sinus maxillaire [3, 17], 4) abord coronal et de la paroi orbitaire latérale [12], 5) un geste chirurgical guidé par la radiologie interventionnelle et CT scanner [17], 6) une fixation de vis de fixation intermaxillaire sur la dent de sagesse et traction [18], ou 7) un abord intra-oral sous anesthésie locale [10,19].

Il existe également des complications associées à la chirurgie de la fosse infra-temporale: importante hémorragie de l'artère maxillaire, lésion neurologique du nerf maxillaire, apparition de diplopie [16]. Au niveau de la fosse ptérygopalatine un déplacement plus postérieur de la dent initialement déplacée peut provoquer un trismus, un œdème pharyngé latéral, de l'hypoesthésie, proptose de la paupière, diplopie, douleur et obstruction nasale [19]. Ainsi, certains auteurs proposent, au lieu d'un traitement chirurgical secondaire, plutôt le suivi clinique et radiologique en espérant une descente spontanée de la dent de sagesse et une récupération de la dent plus facile dans un second temps [3].

Dans le cas présent, nous décrivons un déplacement accidentel du germe de la dent de sagesse dans l'espace jugal antérieur.

\section{Cas clinique}

Une jeune patiente caucasienne de sexe féminin, âgée de 15 ans, sans antécédents médicaux-chirurgicaux, a été adressée par l'orthodontiste traitant auprès d'un chirurgien Oto-rhino-laryngologue (ORL) pour un avis concernant les dents de sagesse. Suite à l'anamnèse, à l'examen clinique et sur base d'une radiographie panoramique (Figure 2) le chirurgien ORL a décidé de procéder à la germectomie des 4 dents de sagesse, sous anesthésie générale, en septembre 2020. 


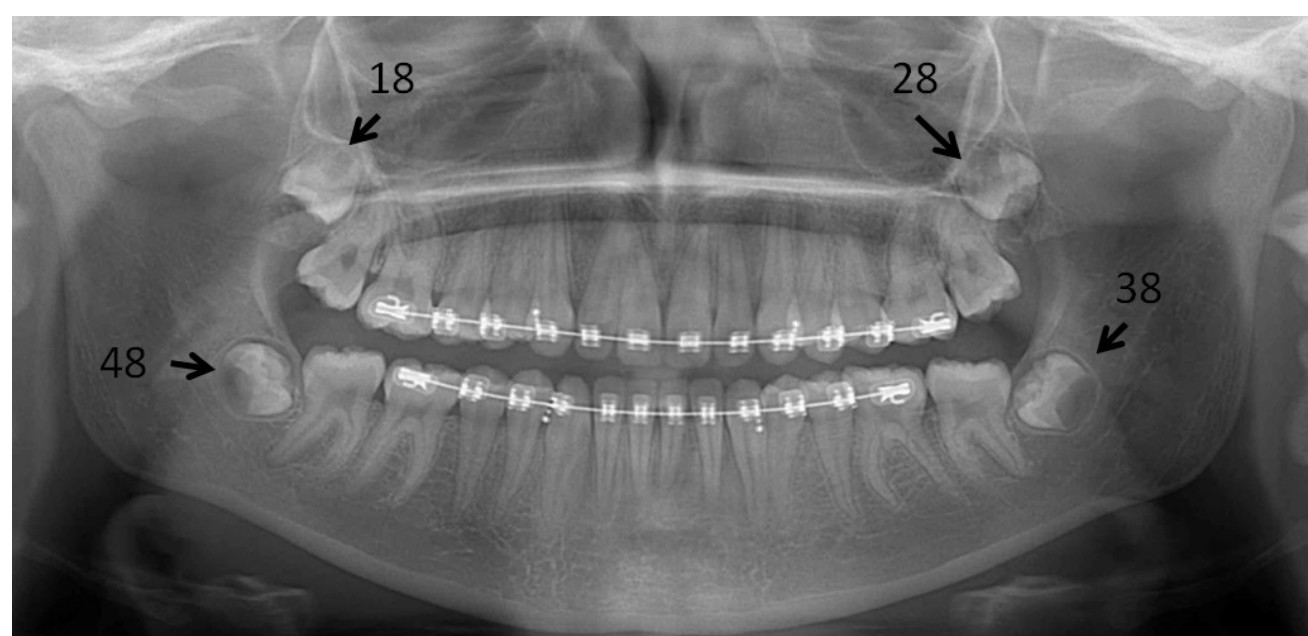

Fig. 2. Radiographie panoramique, janvier 2020. Germes de dents de sagesse $(18,28,38,48)$ profondément incluses, à distance des dents $17 / 27 / 37 / 47$.

Après la période de convalescence post opératoire, la patiente a signalée l'apparition de masse jugale gauche gênant la mastication et provoquant des morsures fréquentes intra-orales. Le chirurgien ORL, d'après la patiente, n'a pas souhaité intervenir. La patiente s'est ensuite adressée à son médecin généraliste traitant qui a prescrit un examen d'échographie de la région parotidienne gauche, dans le but d'exclure ou de confirmer une atteinte parotidienne gauche. Cependant une calcification a été détectée dans la région correspondante à la masse jugale gauche signalée par la patiente. La patiente fut ensuite adressée par le médecin généraliste traitant au dentiste pour un 2ème avis, en décembre 2020, où une imagerie cone beam computed tomography (CBCT) a été prescrite (Planmeca Promax 3D Mid, $10 \mathrm{mAs}, 90 \mathrm{Kvp}, 12.06 \mathrm{~s}$ de temps d'acquisition, taille de champ de vue: $8 \mathrm{~cm}$ de diamètre x $8 \mathrm{~cm}$ de hauteur, DAP: $1177 \mathrm{mGy} / \mathrm{cm}^{2}$, épaisseur de coupe de $200 \mu \mathrm{m}$ ). Le CBCT (Figures 3, 4) a révélé la présence d'un germe dentaire (dent $\mathrm{n}^{\circ} 28$ ) dans le vestibule jugal gauche, à hauteur de la dent $n^{\circ} 26$, correspondant dans sa localisation à l'examen clinique de la masse jugale gauche signalée par la patiente. Par ailleurs, deux traits de fracture étaient présents dans la racine distovestibulaire de la dent $n^{\circ} 27$ (Figure 4). Un fragment de germe de la dent $n^{\circ} 38$ était toujours présent au fond de l'alvéole d'extraction de la dent n 38 (Figure 5). 


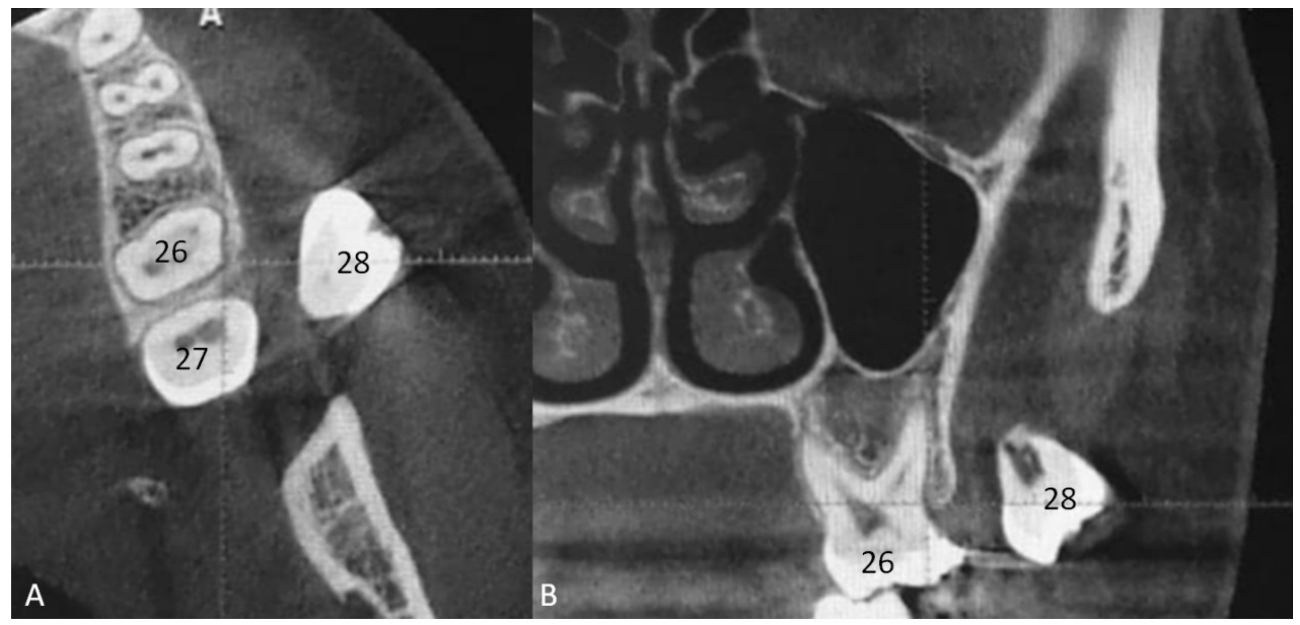

Fig. 3. A. Vue axiale du maxillaire supérieur. Germe de la dent 28 se trouve dans le vestibule, en regard de la dent 26. B. Vue frontale du maxillaire supérieur. Germe de la dent 28 se trouve dans le vestibule jugal en vestibulaire de la dent 26.

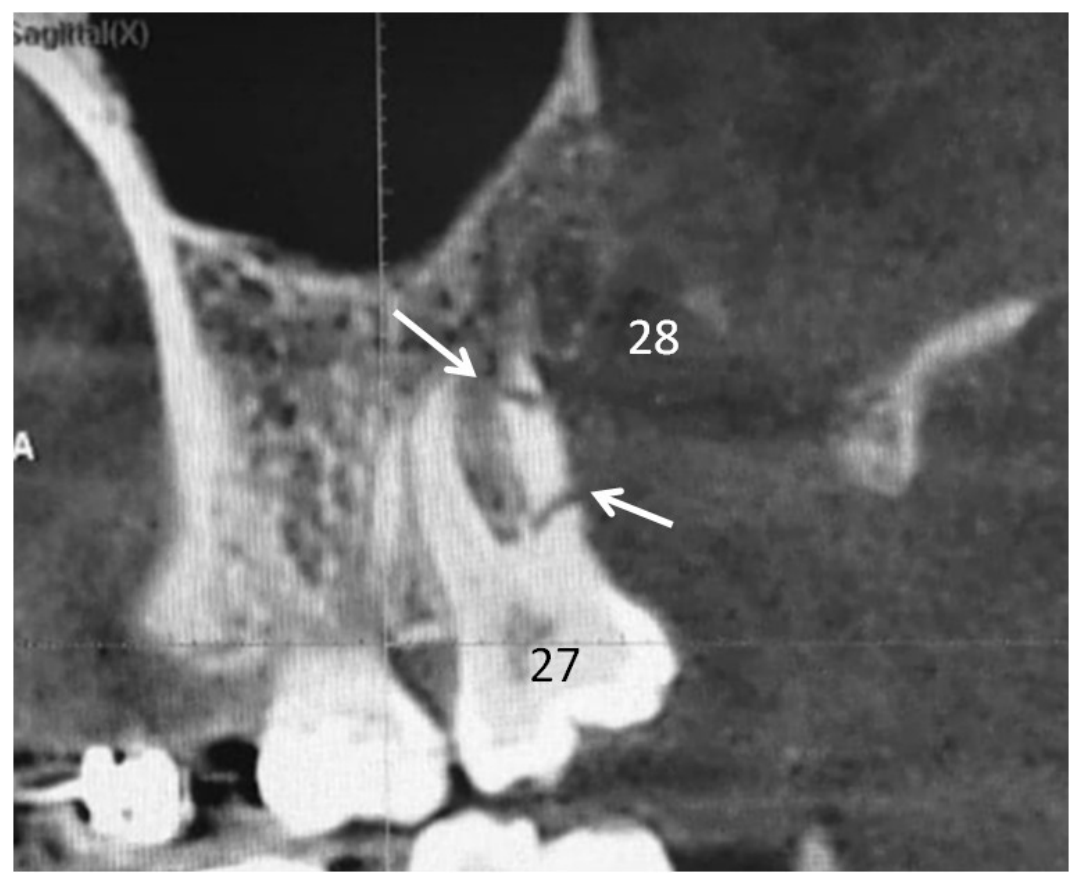

Fig. 4. Flèches: 2 fractures dans la racine disto-vestibulaire de la dent 27. Alvéole vide du germe de la dent 28. 
Fig. 5. A. Vue axiale de la mandibule gauche. Flèche: fragment de la dent 38 dans l'alvéole d'extraction. B. Vue coronale de la mandibule et du maxillaire supérieur gauche. Flèche: fragment de la dent 38 dans l'alvéole d'extraction. C. Vue sagittale de la mandibule et du maxillaire supérieur gauche. Flèche: fragment de la dent 38 dans l'alvéole d'extraction. Flèche avec tirets: canal du nerf alvéolaire inférieur gauche avec préservation de la corticale et proximité avec le fragment de la dent 38 dans l'alvéole d'extraction.

La patiente a été opérée en urgence sous sédation intraveineuse et sous anesthésie locale. Une incision sous l'orifice de canal de Sténon gauche a été effectuée et les tissus mous ont été disséqués. La coque entourant la dent $\mathrm{n}^{\circ} 28$ a été mise en évidence, incisée, et la dent $n^{\circ} 28$ a été est extraite. Au contrôle post opératoire la fonction salivaire parotidienne gauche était bien maintenue.

\section{Discussion}

L'accès ouvert aux informations cliniques dans le domaine des déplacement accidentels des dents de sagesse maxillaires reste extrêmement restreint. Tous les articles du Pubmed étaient payants et leurs abstracts représentaient la seule source de renseignement gratuit pour le praticien privé. Les 4 articles de DOAJ étaient gratuits pour les lecteurs. Par contre, tous ces 4 journaux demandaient des frais de publication imputées aux auteurs, qui oscillaient entre 200 USD (Dental Research Journal) et $1690 £$ par article (BMC Oral Health).

La seule source d'iconographie en accès libre provenait donc des journaux de DOAJ. 7 figures CBCT (dont 1 avec flèches) étaient accessibles pour expliquer le déplacement de la dent de sagesse dans la fosse infra-temporale et 2 figures CT sans flèches étaient disponibles pour l'évaluation de la dent de sagesse repoussée dans la fosse ptérygopalatine. Il n'existe donc pas d'illustration accessible gratuitement pour tous les autres voies de déplacements accidentels des dents de sagesse supérieurs imagées par le CT scan ou par le CBCT. Les images sans flèches sont adressées à un public spécialiste et les images avec flèches à tout public [20]. Une seule image 
CBCT avec flèche est disponible en accès ouvert pour évaluer la position de la dent de sagesse dans la fosse infra-temporale [18].

Le déplacement jugal antérieur de la dent de sagesse au maxillaire supérieur n'a pas encore été décrit. Les symptômes associés différaient des localisations des dents de sagesse déplacés accidentellement vers postérieur par la présence de masse jugale antérieure empêchant la mastication.

Les descriptions de déplacements jugaux concernent le passage de la dent de sagesse vers postérieur, en supérieur des insertions du muscle buccinateur et son passage dans le corps adipeux de la joue [6,9]. Dans le cas présent pour obtenir un déplacement antérieur jugal de la dent, l'effort de l'instrument de luxation a dû être appliqué sur le germe dentaire de postérieur vers antérieur, ce qui est contraire à la luxation standard de antérieur vers postérieur et vers vestibulaire. Par ailleurs, l'effort a dû être suffisamment puissant pour que le germe fracture au passage la racine disto-vestibulaire de la dent 27 (Figure 4).

Enfin, afin d'éviter ce type de complication, un lambeau d'exposition de la dent ainsi qu'une ostéctomie de la paroi osseuse vestibulaire devrait permettre sa visualisation avant de débuter sa luxation [9]. La luxation devrait être réalisée avec l'élévateur avec des mouvements de luxation de la dent d'antérieur vers postérieur et vers vestibulaire [9]. Une rugine devrait être placée en distal pour empêcher le germe de la dent de sagesse de glisser vers distal et crânial [9]. Si le déplacement accidentel survient, il faut prévenir le patient et nous préconisons également la réalisation, au minimum, d'une radiographie panoramique post-opératoire et d'un CBCT en cas de confirmation de déplacement accidentel pour pouvoir préciser la localisation du germe.

- Remerciements: aucun

- Sources de financement: cette étude n'a reçu aucun soutien financier.

- Conflits d'intérêt: Pr R. Olszewski est l'Editeur-en-Chef de la revue NEMESIS. Mr S. Fathi déclare qu'il n'a pas de conflit d'intérêt lié à cette étude.

- Approbation éthique: Ce rapport de cas n'a pas nécessité d'accord de comité d'éthique

- Consentement éclairé: Ce rapport de cas n'a pas nécessité de consentement éclairé de la part du patient. Toutes les images sont anonymisées. Aucune donnée qui permettrait d'identifier le patient n'a été fournie. 


\section{Contribution des auteurs}

\begin{tabular}{|l|l|}
\hline Auteur & Contribution \\
\hline Fathi S & $\begin{array}{l}\text { Découverte du cas, Documentation, } \\
\text { Investigation, Rédaction, } \\
\text { Correction, Relecture }\end{array}$ \\
\hline Olszewski R & $\begin{array}{l}\text { Documentation, Investigation, Rédaction, } \\
\text { Correction, Relecture, Supervision, }\end{array}$ \\
\hline
\end{tabular}

\section{References}

1. Baba Aissa M. Complications lors des avulsions des dents de sagesse : comment les éviter. Sciences du Vivant [q-bio]. 2017. ffdumas-01659099f HAL Id: dumas01659099 https://dumas.ccsd.cnrs.fr/dumas-01659099

2. Christiaens I, Reychler H. Complications after third molar extractions: retrospective analysis of 1213 teeth. Rev Stomatol Chir Maxillofac 2002;103:269 274.

3. Bozkurt P, Erdem E. Management of upper and lower molars that are displaced into the neighbouring spaces. Br J Oral Maxillofac Surg 2017;55:e49-e52.

4. Battisti A, Priore P, Giovannetti F, Barbera G, D'Alessandro F, Valentini V. Rare complication in third maxillary molar extraction: dislocation in infratemporal fossa. J Craniofac Surg 2017;28:1784-1785.

5. Sencimen M, Gülses A, Secer S, Zerener T, Özarslantürk S. Delayed retrieval of a displaced maxillary third molar from infratemporal space via trans-sinusoidal approach: a case report and the review of the literature. Oral Maxillofac Surg 2017;21:1-6.

6. Ohba S, Nakatani Y, Kakehashi H, Asahina I. The migration pathway of an extracted maxillary third molar into the buccal fat pad. Odontology. 2014;102:33942.

7. Lee D, Ishii S, Yakushiji N. Displacement of maxillary third molar into the lateral pharyngeal space. J Oral Maxillofac Surg 2013;71:1653-1657. 
8. Selvi F, Cakarer S, Keskin C, Ozyuvaci H. Delayed removal of a maxillary third molar accidentally displaced into the infratemporal fossa. J Craniofac Surg 2011;22:1391-1393.

9. Kocaelli H, Balcioglu HA, Erdem TL Displacement of a maxillary third molar into the buccal space: anatomical implications apropos of a case. Int $\mathrm{J}$ Oral Maxillofac Surg 2011;40:650-653.

10. Sverzut CE, Trivellato AE, Sverzut AT, de Matos FP, Kato RB. Removal of a maxillary third molar accidentally displaced into the infratemporal fossa via intraoral approach under local anesthesia: report of a case. J Oral Maxillofac Surg 2009;67:1316-1320.

11. Dimitrakopoulos I, Papadaki M. Displacement of a maxillary third molar into the infratemporal fossa: case report. Quintessence Int 2007;38:607-610.

12. Sverzut CE, Trivellato AE, Lopes LM, Ferraz EP, Sverzut AT. Accidental displacement of impacted maxillary third molar: a case report. Braz Dent J 2005;16:167-170.

13. Durmus E, Dolanmaz D, Kucukkolbsi H, Mutlu N. Accidental displacement of impacted maxillary and mandibular third molars. Quintessence Int 2004;35:375-377.

14. Gulbrandsen SR, Jackson IT, Turlington EG. Recovery of a maxillary third molar from the infratemporal space via a hemicoronal approach. J Oral Maxillofac Surg 1987;45:279-282.

15. Iwai T, Chikumaru H, Shibasaki M, Tohnai I. Safe method of extraction to prevent a deeply-impacted maxillary third molar being displaced into the maxillary sinus. Br J Oral Maxillofac Surg 2013;51:e75-76.

16. Roshanghias K, Peisker A, Zieron JO. Maxillary tooth displacement in the infratemporal fossa. Dental Research Journal 2016;13:373-375.

17. Lutz JC, Cazzato RL, Le Roux MK, Bornert F. Retrieving a displaced third molar from the infratemporal fossa: case report of a minimally invasive procedure. BMC Oral Health 2019;19:149.

18. Dallazen E, Almeida Carvalho V, Hochuli-Vieira E, Statkievcz C, PereiraStabile CL, Vitti Stabile GA, Lecher Borges HO. Late removal of an upper third molar displaced into the infratemporal space using an intermaxillary fixation screw: a case report. Research, Society and Development 2020;9:e129119457. 
2

19. Özer N, Üçem F, Saruhanoğlu A, Yilmaz S, Tanyeri H. Removal of a maxillary third molar displaced into pterygopalatine fossa via intraoral approach. Case Reports in Dentistry 2013;ID 392148 https://doi.org/10.1155/2013/392148

20. Olszewski R, Hebda A. Accessibility to the knowledge on anatomical variations from dentomaxillofacial CBCT. Nemesis 2020;10:1-19. 